\title{
Analysis of clinical patient-specific pre-treatment quality assurance with the new helical tomotherapy platform, following the AAPM TG-218 report
}

\author{
Marco Fusella ${ }^{1}$, Samuele Cavinato ${ }^{1,2}$, Alessandra Germani ${ }^{1}$, Marta Paiusco ${ }^{1}$, Nicola Pivato ${ }^{1}$, \\ Marco Andrea Rossato ${ }^{1}$, Anthony Scott ${ }^{3}$ and Alessandro Scaggion ${ }^{1 *}$ (i)
}

\begin{abstract}
Purpose: This study presents patient-specific quality assurance (QA) results from the first 395 clinical cases for the new helical TomoTherapy ${ }^{\circledR}$ platform (Radixact) coupled with dedicated Precision TPS.

Methods: The passing rate of the Gamma Index (GP\%) of 395 helical QA of patient-specific tomotherapy, acquired with ArCCHECK, is presented, analysed and correlated to various parameters of the plan. Following TG-218 recommendations, the clinic specific action limit $\left(A L_{c s}\right)$ and tolerance limit $\left(T L_{c s}\right)$ were calculated for our clinic and monitored during the analysed period.

Results: The mean values ( \pm 1 standard deviation) of GP\% (3\%/2 mm) (both global and local normalization) are: $97.6 \%$ and $90.9 \%$, respectively. The proposed $\mathrm{AL}_{\mathrm{cs}}$ and $\mathrm{TL}_{\mathrm{cs}}$, fter a period of two years' process monitoring are $89.4 \%$ and $91.1 \%$ respectively.

Conclusions: The phantom measurements closely match the planned dose distributions, demonstrating that the calculation accuracy of the new Precision TPS and the delivery accuracy of the Radixact unit are adequate, with respect to international guidelines and reports. Furthermore, a first correlation with the planning parameters was made. Action and tolerance limits have been set for the new Radixact Linac.
\end{abstract}

Keywords: TomoTherapy, Quality assurance, Pre-treatment quality assurance, IMRT QA

\section{Introduction}

In early 2018 a new version of the TomoTherapy ${ }^{\circledR}$ platform was commercially released (Radixact, Accuray, Sunnyvale, CA). This new machine is characterized by a higher dose rate of $1000 \mathrm{cGy} / \mathrm{min}$, improved jaw dynamics, faster ring rotation (up to 10 rotations per minute), couch movement with separated axis and a couch

${ }^{*}$ Correspondence: alessandro.scaggion@iov.veneto.it

1 Medical Physics Department, Veneto Institute of Oncology IOV-IRCCS, Via Gattamelata 64, 35128 Padova, Italy

Full list of author information is available at the end of the article catcher to reduce couch sag effect (to less than $2 \mathrm{~mm}$ ) toward the gantry. The new platform comes with a redesigned version of the TPS: Precision TPS v1.0.02 which incorporates the graphics processing unit (GPU) based optimizer, VoLO ${ }^{\mathrm{TM}}$ and a Collapsed Cone Convolution Superposition dose calculation algorithm [1, 2].

This new system grants the capability of faster treatments and more freedom in the combination of the modulation factor, jaw width, pitch and gantry period. Thanks to these advanced features, the use of this system opens to the possibility of achieving more modulated and complex plans. original author(s) and the source, provide a link to the Creative Commons licence, and indicate if changes were made. The images or other third party material in this article are included in the article's Creative Commons licence, unless indicated otherwise in a credit line to the material. If material is not included in the article's Creative Commons licence and your intended use is not permitted by statutory regulation or exceeds the permitted use, you will need to obtain permission directly from the copyright holder. To view a copy of this licence, visit http://creativecommons.org/licenses/by/4.0/. The Creative Commons Public Domain Dedication waiver (http://creativeco mmons.org/publicdomain/zero/1.0/) applies to the data made available in this article, unless otherwise stated in a credit line to the data. 
The long term dosimetric stability of this new delivery system has already been studied and reported by Smilowitz [3] and the new TPS version has been validated by Chen [2]. In order to thoroughly assess the new capability of the system, a detailed and complete analysis on a large database of clinical plans is also needed.

At our department, a Radixact unit machine has become clinical in June 2018 and since the first clinical plan a detailed QA program has been implemented following the recommendations of AAPM TG-218 [4]. Approximately 395 clinical helical plans were delivered and measured with ArcCHECK ${ }^{\mathrm{TM}}$ (Sun Nuclear, Melbourne, FL). Measured and calculated dose distributions were compared by means of 2D gamma analysis.

Within this work, we propose the clinic and machine specific tolerance limit $\left(\mathrm{TL}_{\mathrm{cs}}\right)$ and action limit $\left(\mathrm{AL}_{\mathrm{cs}}\right)$, obtained through statistical process control techniques, to be adopted for the new Radixact platform coupled to the Precision TPS v1.0.02. We also analyse and report which planning parameters are more related to QA failures.

\section{Materials and methods Database}

Between June 2018 and October 2020 a total of 395 treatments have been planned with the Precision TPS v1.0.02 using the GPU-based optimizer VoLO ${ }^{\mathrm{TM}}$, which incorporates the Collapsed Cone Convolution Superposition dose calculation algorithm [1,2]. The treatments were related to a large number of different sites and diseases; therefore, within the scope of this work they have been grouped in the following categories: abdomen (28 pts), brain (32 pts), head and neck (77 pts), lungs (71 pts), pelvis (84 pts), prostate (52 pts) and others (41 pts). The dose per fraction $(\mathrm{D} / \mathrm{fr})$ ranged from 1.6 to $5 \mathrm{~Gy}$.

\section{Plans characteristics}

To characterize the plans, the following parameters were collected: actual modulation factor (MF), pitch, field width (FW), gantry period, total delivery time, couch speed, couch travel distance and TTDF (ratio between the treatment time and the prescribed dose per fraction). Moreover, some descriptors of the leaf open time (LOT) distribution were collected: minimum (min-LOT), mean (mean-LOT), maximum (max-LOT) and LOT standard deviation (SD-LOT). All treatments utilized dynamic jaws [5].

\section{QA delivery and analysis}

All the patient-specific QA measurements were collected with ArcCHECK $^{\mathrm{TM}}$ (Sun Nuclear, Melbourne, FL) and performed without the $15 \mathrm{~cm}$ diameter homogeneous PMMA plug. The plans were recomputed on the synthetic ArcCHECK ${ }^{\mathrm{TM}} \mathrm{CT}$ provided by the vendor with an imposed density of $1.1836 \mathrm{~g} / \mathrm{cm}^{3}$ following manufacturer's recommendations. Acquisition and analysis were performed with Sun Nuclear SNC Patient ${ }^{\mathrm{TM}}$ version 6.7. The absolute dose calibration of $\operatorname{ArcCHECK}^{\mathrm{TM}}$ was periodically controlled against a calibrated farmer-type ionisation chamber. Before each measurement session, the output of the Radixact unit was checked using the cheese phantom and ionization chambers, and a correction applied to the $\operatorname{ArcCHECK}^{\mathrm{TM}}$ analysis. QA plans were computed in high resolution mode which grants a 1.87 mm spaced dose grid.

The QA plan was computed placing the centre of the planning PTV at the machine's isocenter. This procedure reduces the possibility for high dose areas to fall at the border of the ArcCHECK ${ }^{\mathrm{TM}}$ limiting the dependence of the gamma passing rate $(\mathrm{GP} \%)$ on the maximum calculated dose [6].

According to the AAPM TG-218 report, the dose comparison was performed through $2 \mathrm{D}$ gamma analysis using a $10 \%$ dose threshold as well as $3 \%$ dose difference (DD) and $2 \mathrm{~mm}$ distance-to-agreement (DTA) criteria. In order to compare our results with previous works [5-8], $3 \% / 3 \mathrm{~mm}$ gamma analysis was also collected. Both local and global normalizations were used.

\section{QA program}

Following the AAPM TG-218 report, the clinical deliverability of a plan was evaluated on the basis of the universal tolerance limit (TL) and action limit (AL), which are $95 \%$ and $90 \%$ respectively, on the GP\% computed with $(3 \%, 2 \mathrm{~mm})$, global normalization criteria.

Statistical process control techniques recommended in TG-218 have been employed to define a clinic specific $\mathrm{TL}_{\mathrm{cs}}$ and $\mathrm{AL}_{\mathrm{cs}}[4]$ as follows:

$$
\begin{aligned}
& A L_{c s}=100-3 \sqrt{\sigma^{2}+(\underline{x}-100)^{2}} \\
& T L_{c s}=\underline{x}-2.660 \mathrm{mR}
\end{aligned}
$$

where $\underline{x}$ is the GP\% average over the investigation period, $\sigma$ is its standard deviation and $m R$ the moving average. These values were computed on the first $40 \mathrm{col}$ lected measurements. The IMRT treatment process has been monitored and investigated by periodically computing these values to verify whether the process was under control. For the subsequent evaluations, periods of approximately 6 months were considered. Only clinically deliverable plans, those fulfilling GP\% (3\%G, $2 \mathrm{~mm})>$ $90 \%$, were considered in this analysis and $95 \%$ confidence intervals on $\mathrm{TL}_{\mathrm{cs}}$ and $\mathrm{AL}_{\mathrm{cs}}$ have been computed through a bootstrap approach with 10,000 samples $[9,10]$. 


\section{Statistical analysis}

To spot possible differences of GP\% among the different treatment sites a Kruskal-Wallis test was performed followed by a post-hoc analysis where Bonferroni correction was applied [11].

$\mathrm{N}$-way analysis of variance (n-way ANOVA) was performed to spot the influence of the planning parameters on GP\% [12]. Only parameters that strictly bore no correlations among themselves were included in the test $(p>$ 0.05 and/or Pearson's $r<0.8)[13,14]$.

All tests were performed using MATLAB ${ }^{\circledR}$ R2020b (MathWorks, Inc., Natick, MA, USA). All the $p$-values reported are two-sided and $p<0.05$ is considered as statistically significant.

\section{Results}

Over the entire collection period, only 10 out of 385 plans failed to meet the universal AL defined by the TG-218 report. These failing plans were re-planned to meet the universal AL and added to the original set, thus resulting in a total of 395 plans that were actually measured. Figure 1 depicts the whole population of collected QA results.

The measured 3\%/2 mm GP\% (both global and local normalizations) of the whole population $(\mathrm{n}=395)$ was distinguished among treatment sites and the information is presented in Fig. 2. Data include both clinically and

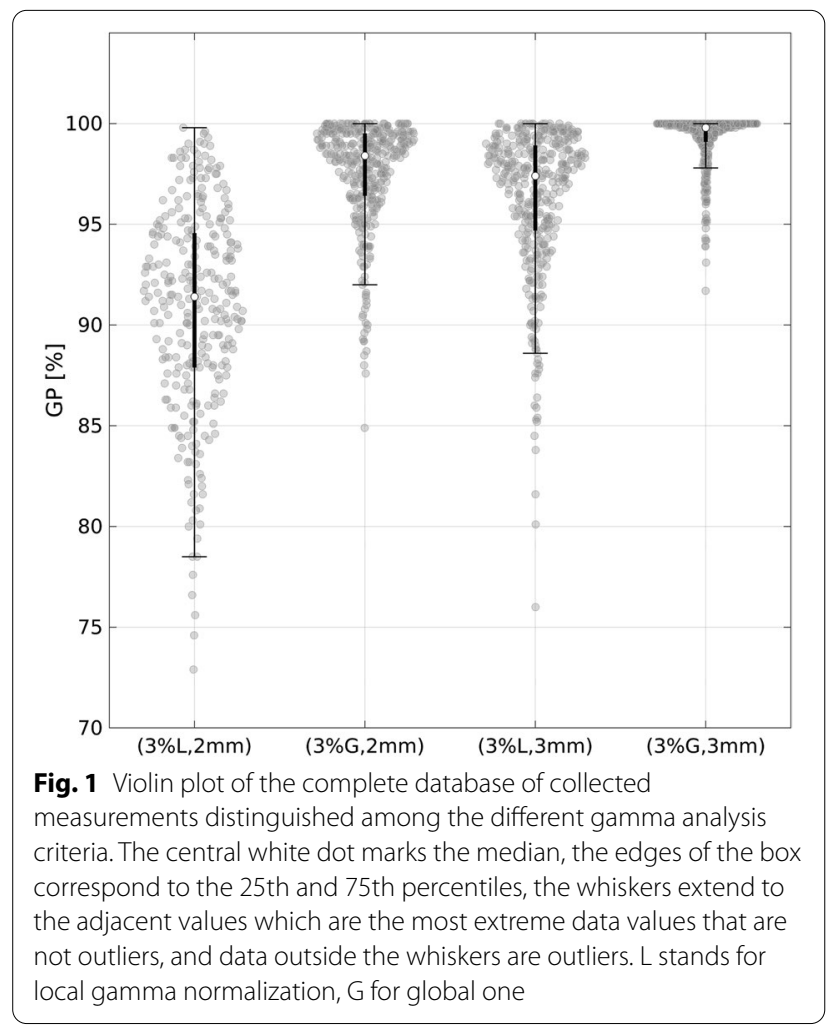

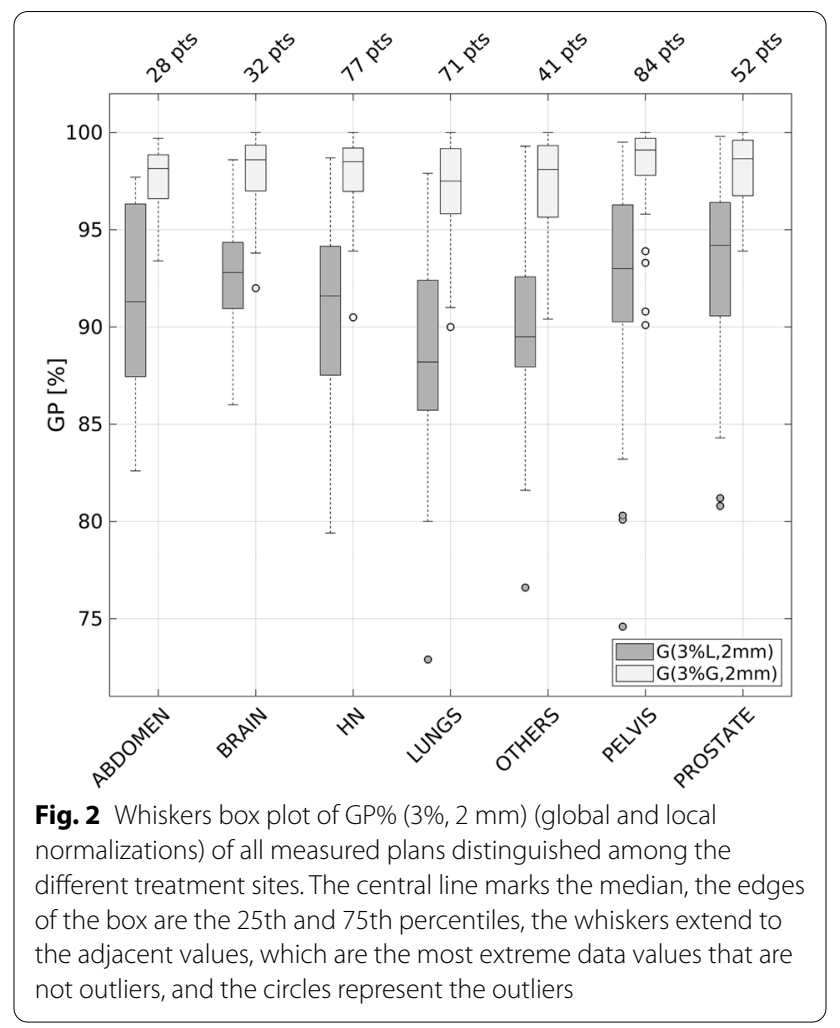

non-clinically $(\mathrm{GP} \%(3 \% \mathrm{G}, 2 \mathrm{~mm})<90 \%)$ delivered plans. The Kruskal-Wallis test confirmed that some differences among the groups does exist ( $p$-value $<0.001)$. The posthoc test showed that these differences are statistically significant only when the pelvis plans are compared to abdomen, lungs and others groups.

Table 1 reports the distribution of the collected planning parameters for our database of plans.

Table 1 Collected planning parameters for the entire population of 395 collected QA deliveries

\begin{tabular}{lll}
\hline & Average \pm SD & Range [min; max] \\
\hline Dose/Fraction [cGy] & $219.8 \pm 62.6$ & {$[160.0 ; 500.0]$} \\
MF & $1.68 \pm 0.28$ & {$[1.10 ; 2.79]$} \\
Pitch & $0.39 \pm 0.06$ & {$[0.12 ; 0.48]$} \\
Gantry Period [s] & $20.1 \pm 7.3$ & {$[11.8 ; 52.8]$} \\
Total Treatment Time [s] & $299.7 \pm 141.0$ & {$[66.3 ; 1241.3]$} \\
TTDF [s/cGy] & $1.41 \pm 0.69$ & {$[0.36 ; 5.43]$} \\
min-LOT [ms] & $18.2 \pm 1.1$ & {$[18.0 ; 36.4]$} \\
mean-LOT [ms] & $236.3 \pm 82.7$ & {$[57.8 ; 617.1]$} \\
max-LOT [ms] & $391.4 \pm 144.0$ & {$[123.2 ; 1034.2]$} \\
SD-LOT [ms] & $107.9 \pm 44.2$ & {$[21.1 ; 316.1]$} \\
Couch speed [mm/s] & $0.56 \pm 0.20$ & {$[0.11 ; 1.84]$} \\
Couch travel distance [mm] & $160.2 \pm 88.9$ & {$[31.1 ; 895.2]$} \\
FW $(10$ mm/25 mm/50 mm) & $2.1 \% / 92.0 \% / 5.9 \%$ & \\
\hline
\end{tabular}




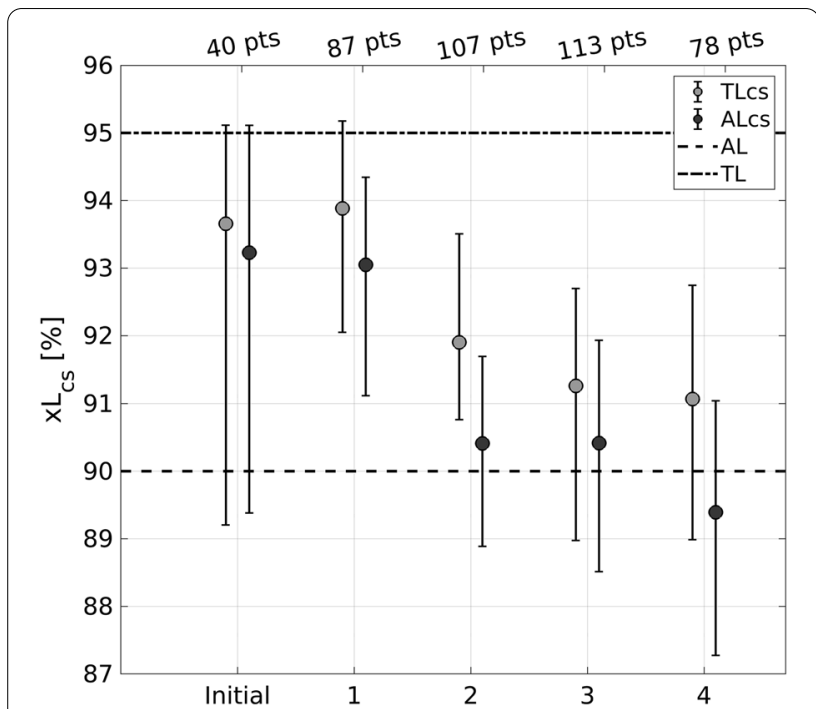

Fig. 3 Evaluation of the $A L_{c s}$ and $T L_{c s}$. The circles mark the obtained values while the whiskers extend to the $95 \%$ confidence interval

Figure 3 reports the periodical evaluation of the $\mathrm{AL}_{\mathrm{cs}}$ and $\mathrm{TL}_{\mathrm{cs}} ;$ non-clinical plans are not included. The first 40 plans used for the first calculations (as proposed in TG-218), are also included in period "1".

Among the first 40 planned treatments, the calculated $\mathrm{AL}_{\mathrm{cs}}$ and $\mathrm{TL}_{\mathrm{cs}}$ are, respectively, $93.2 \%$ and $93.7 \%$, with all the plans satisfying GP\% $(3 \% \mathrm{G}, 2 \mathrm{~mm})>93.3 \%$. The final computation of $\mathrm{AL}_{\mathrm{cs}}$ and $\mathrm{TL}_{\mathrm{cs}}$ on the last set of 78 clinical plans yielded $\mathrm{AL}_{\mathrm{cs}}=89.4 \%$ and $\mathrm{TL}_{\mathrm{cs}}=91.1 \%$.

A Kruskal-Wallis test has been performed to compare $\mathrm{AL}_{\mathrm{cs}}$ and $\mathrm{TL}_{\mathrm{cs}}$ samples of period 2, 3 and 4 and no statistically significant differences exist. In these three periods not even a single plan lies below the estimated $\mathrm{AL}_{\mathrm{cs}}$, following the recommendations of TG-218, these evidences allow to state that the process is settled and under control. Using data from these 3 periods a n-way ANOVA was performed to detect which parameters might predict QA failures are summarized for both local and global normalizations of GP\% with $3 \% / 2 \mathrm{~mm}$ criteria. Results are given in Table 2.

In Table 2, the results of the n-way ANOVA performed The analysis has been conducted for the data of the last three periods, where the process seems to become settled and under control (Fig. 3).

\section{Discussion}

In this work, we assessed the performances of the TomoTherapy ${ }^{\circledR}$ platform coupled with a new dedicated TPS in terms of the gamma index passing rate measured with a $3 \mathrm{D}$ dosimeter, extending the analysis of previous works on earlier versions of TomoTherapy ${ }^{\circledR}$ and associated TPS $[7,8]$. The entire scope of work was performed following
Table 2 Results of the n-way ANOVA test on GP\% (3\%, 2 mm) with respect to all the collected planning parameters in terms of $p$-values

\begin{tabular}{lll}
\hline & $\mathbf{( 3 \% \mathbf { L } , \mathbf { 2 ~ } \mathbf { ~ m } )}$ & $\mathbf{( 3 \% \mathbf { G } , \mathbf { 2 ~ } \mathbf { m m } )}$ \\
\hline Site & $\underline{0.002}$ & 0.063 \\
MF & 0.294 & 0.199 \\
TTDF & $\underline{0.007}$ & 0.373 \\
mean-LOT & 0.642 & 0.437 \\
max-LOT & 0.804 & 0.859 \\
Pitch & 0.884 & 0.659 \\
Gantry period & 0.820 & 0.841 \\
Couch speed & 0.189 & 0.055 \\
\hline
\end{tabular}

Underlined values mark significant tests

AAPM TG-218 recommendations. We considered the GP\% metric with $3 \% / 2 \mathrm{~mm}$ criteria, analysing also the impact of local and global normalizations. Our results showed that the mean GP\% $(3 \% \mathrm{G} / 2 \mathrm{~mm})$ was $97.6 \pm$ $2.6 \%$ with some differences arising among the different sites used to stratify the sample.

Any previous study on the TomoTherapy ${ }^{\circledR}$ unit has not reported results using the GP\% metric defined by AAPM TG-218. For a comparison with existing literature we used the GP\% with $3 \% / 3 \mathrm{~mm}$ criteria. The results here reportedly outperformed those obtained with different versions of tomotherapy machines and the same 3D dosimeter $\left(\mathrm{ArcCHECK}^{\mathrm{TM}}\right)$ in terms of GP\%. We measured systematically higher mean values with respect to Bresciani [7], Binny [15] and Yue [16]: 99.2\% (SD 1.3\%) vs. $96.1 \%$ (SD 4.4\%), 95.9\% (SD 2.9\%) and 97.5\% (SD $2.7 \%)$, respectively.

Applying the statistical process control techniques proposed by the Task Group, we periodically computed the centre-specific action level and tolerance limit (see Fig. 3). The first evaluation yielded values comparable to the universal ones indicated in the report $(93.7 \%$ and $93.2 \%$, versus $95 \%$ and $90 \%$ respectively). The periodical evaluation of the two control limits showed that a change occurred in the process between the first and the second re-evaluation. In fact, both $\mathrm{TL}_{\mathrm{cs}}$ and $\mathrm{AL}_{\mathrm{cs}}$ decreased and remained approximately constant afterwards (Fig. 3). This probably happened since the start of 2019 and after six months of initial training, new treatments have been introduced into the clinical practice (e.g. mesotheliomas, craniospinal irradiations, Hodgkin's and non-Hodgkin's lymphomas) that can be considered as inherently more complex; moreover, moving up the learning curve, the planners have begun to achieve increasingly complex and modulated dose distribution; thus probably inducing a decrease of treatment deliverability. All 
this sources of variation forced to follow TG-218 recommendations, going through a periodically monitoring of the process, investigating the GP\% over a long period.

To spot possible planning parameters closely related to QA results, and their trend, a n-way ANOVA test was performed (see Table 2). Only the treatment site and the TTDF (treatment time divided by the prescribed dose per fraction) were highlighted as possible predictors of QA failures, only when GP\% $(3 \% \mathrm{~L}, 2 \mathrm{~mm})$ is considered. In fact, the TTDF can be considered as a simple indicator of complexity, since it is somehow related to the longitudinal extension of the target volume and to the pitch. A similar behaviour was recently reported by Santos et al. [17].

Previous studies reported the effect of mean-LOT, max-LOT, MF, pitch and gantry period on plan deliverability $[6,7,15,18]$. In our database, none of the collected parameter can be considered relevant when the $\mathrm{GP} \%(3 \% \mathrm{G}, 2 \mathrm{~mm})$ is used, and this might be due to different reasons. First of all, the presence of the TTDF might mask all the other parameters, since mathematical relationships hold between the TTDF and most of the aforementioned parameters. Secondly, our planning practice strictly follows the recommendation of the Accuray planning manual [1] which suggests to prepare plans with max-LOT higher than $241 \mathrm{~ms}$, and mean-LOT higher than $100 \mathrm{~ms}$. Moreover, approximately $95 \%$ of our plans show the MF within 1.4 and 2.5, which Binny et al. [18] have previously shown to be associated to acceptable deliverability.

Because no dependence from anatomical site has been found for $\mathrm{GP} \%$ ( $3 \% \mathrm{G} / 2 \mathrm{~mm}$ ), it is possible to use for all treatments the same $\mathrm{AL}_{\mathrm{cs}}$ and $\mathrm{TL}_{\mathrm{cs}}$ here reported. When the more stringent local criteria is considered a significant difference among the anatomical site arouse. In this case group-specific $\mathrm{AL}_{\mathrm{cs}}$ and $\mathrm{TL}_{\mathrm{cs}}$ would have been required and, following AAPM TG-218 recommendation, should be computed.

The assessment of the TPS's performances in regards to complexity of the calculated sinograms is beyond the scope of this work, as well as the detailed analysis of the relationship between GP\% and clinically relevant deviations [6,19-21]. A more detailed analysis on the predictive value of the analysed parameters on QA trend and failures is still ongoing.

The results of this study highlight the capability of the Radixact system to accurately deliver complex dose distributions over a large variety of treatment sites. We set the TL and AL for this new machine to be used as reference or comparison for other centres.

\section{Conclusions}

The results presented in this study suggest that the calculation accuracy of the new Precision TPS and the delivery accuracy of the Radixact unit is adequate, with respect to international guidelines and reports. Following the TG-218 methodology, we calculated both tolerance and action limits for the determination of the clinical deliverability of plans calculated with the new TPS, Accuray Precision, for the new TomoTherapy ${ }^{\circledR}$ version, (Radixact). These values have been monitored over a long period, and can be used as reference or comparison for other centres implementing this new technology.

\section{Abbreviations \\ AL: Action limit; $\mathrm{AL}_{\mathrm{cs}}$ : Center specific action limit; $\mathrm{CT}$ : Computed tomography; DD: Dose difference; DTA: Distance-to-agreement; FW: Field width; GP\%: Gamma-analysis passing rate; LOT: Leaf open time; MF: Actual modulation factor; PTV: Planning target volume; QA: Quality assurance; TG: Task group; TL: Tolerance limit; $\mathrm{TL}_{\mathrm{cs}}$ : Center specific tolerance limit; TPS: Treatment planning system; TTDF: Treatment time over dose per fraction.}

\section{Acknowledgements}

Not applicable.

\section{Authors' contributions}

All the listed authors have contributed to the work, and approved the manuscript for submission. AS and MF take care of preparing the manuscript, SC take care of statistical analysis and figure composition.

\section{Authors' information}

Dr. Marco Fusella, acting as first author of this manuscript acknowledges the following: He did not receive payment or services from a third party (government, commercial, private foundation, etc.) for any aspect of the submitted work. He did not have any financial relationships, during the 36 months prior to submission, a part from the presenting institution. He does not have any patents, whether planned, pending or issued, broadly relevant to the presented work. There are no other relationships or activities that readers could perceive to have influenced, or that give the appearance of potentially influencing, what he wrote in the submitted workBased on the above disclosures Dr. Marco Fusella has nothing to disclose.

Dr. Alessandro Scaggion, acting as corresponding author of this manuscript acknowledges the following: He did not receive payment or services from a third party (government, commercial, private foundation, etc.) for any aspect of the submitted work. He did not have any financial relationships, during the 36 months prior to submission, a part from the presenting institution. He does not have any patents, whether planned, pending or issued, broadly relevant to the presented work. There are no other relationships or activities that readers could perceive to have influenced, or that give the appearance of potentially influencing, what he wrote in the submitted work. Based on the above disclosures Dr. Alessandro Scaggion has nothing to disclose.

Dr. Samuele Cavinato, acting as contributing author of this manuscript acknowledges the following: He did not receive payment or services from a third party (government, commercial, private foundation, etc.) for any aspect of the submitted work. He did not have any financial relationships, during the 36 months prior to submission, a part from the presenting institution. He does not have any patents, whether planned, pending or issued, broadly relevant to the presented work. There are no other relationships or activities that readers could perceive to have influenced, or that give the appearance of potentially influencing, what he wrote in the submitted work. Based on the above disclosures Dr. Samuele Cavinato has nothing to disclose.

RTT Alessandra Germani, acting as contributing author of this manuscript acknowledges the following: He did not receive payment or services from a third party (government, commercial, private foundation, etc.) for any aspect of the submitted work. He did not have any financial relationships, during the 36 months prior to submission, a part from the presenting institution. He does 
not have any patents, whether planned, pending or issued, broadly relevant to the presented work. There are no other relationships or activities that readers could perceive to have influenced, or that give the appearance of potentially influencing, what he wrote in the submitted work. Based on the above disclosures RTT Alessandra Germani has nothing to disclose.

Dr. Marta Paiusco, acting as contributing author of this manuscript acknowledges the following: He did not receive payment or services from a third party (government, commercial, private foundation, etc.) for any aspect of the submitted work. He did not have any financial relationships, during the 36 months prior to submission, a part from the presenting institution. He does not have any patents, whether planned, pending or issued, broadly relevant to the presented work. There are no other relationships or activities that readers could perceive to have influenced, or that give the appearance of potentially influencing, what he wrote in the submitted work. Based on the above disclosures Dr. Marta Paiusco has nothing to disclose.

RTT Nicola Pivato, acting as contributing author of this manuscript acknowledges the following: He did not receive payment or services from a third party (government, commercial, private foundation, etc.) for any aspect of the submitted work. He did not have any financial relationships, during the 36 months prior to submission, a part from the presenting institution. He does not have any patents, whether planned, pending or issued, broadly relevant to the presented work. There are no other relationships or activities that readers could perceive to have influenced, or that give the appearance of potentially influencing, what he wrote in the submitted work. Based on the above disclosures RTT Nicola Pivato has nothing to disclose.

RTT Marco Andrea Rossato, acting as contributing author of this manuscript acknowledges the following: He did not receive payment or services from a third party (government, commercial, private foundation, etc.) for any aspect of the submitted work. He did not have any financial relationships, during the 36 months prior to submission, a part from the presenting institution. He does not have any patents, whether planned, pending or issued, broadly relevant to the presented work. There are no other relationships or activities that readers could perceive to have influenced, or that give the appearance of potentially influencing, what he wrote in the submitted work. Based on the above disclosures RTT Marco Andrea Rossato has nothing to disclose.

Dr. Anthony Scott, acting as contributing author of this manuscript acknowledges the following: He did not receive payment or services from a third party (government, commercial, private foundation, etc.) for any aspect of the submitted work. He did not have any financial relationships, during the 36 months prior to submission, a part from the presenting institution. He does not have any patents, whether planned, pending or issued, broadly relevant to the presented work. There are no other relationships or activities that readers could perceive to have influenced, or that give the appearance of potentially influencing, what he wrote in the submitted work. Based on the above disclosures Dr. Anthony Scott has nothing to disclose.

\section{Funding}

No payment or services from a third party (government, commercial, private foundation, etc.) for any aspect of the submitted work was received by any of the authors.

\section{Availability of data and materials}

Not applicable.

\section{Declarations}

Ethics approval and consent to participate

Not applicable.

\section{Consent for publication}

Not applicable.

\section{Competing interests}

The authors do not have any competing interests.

\section{Author details}

${ }^{1}$ Medical Physics Department, Veneto Institute of Oncology IOV-IRCCS, Via Gattamelata 64, 35128 Padova, Italy. ${ }^{2}$ Dipartimento di Fisica e Astronomia

'G. Galilei', Università degli Studi di Padova, Padova, Italy. ${ }^{3}$ The Abdus Salam
International Centre for Theoretical Physics, Strada Costiera 11, 34151 Trieste, Italy.

\section{Received: 22 September 2021 Accepted: 11 November 2021}

Published online: 22 November 2021

\section{References}

1. Radixact Physics Essential Guide 1060900-ENG A, Acccuray, Version 1.1.X, Date of Revision: 2018-01-02.

2. Chen Q, Lu W, Chen Y, Chen M, Henderson D, Sterpin E. Validation of GPU based TomoTherapy dose calculation engine: TomoTherapy GPU dose validation. Med Phys 2012; 39:1877-86. https://doi.org/10.1118/1.36930 57.

3. Smilowitz JB, Dunkerley D, Hill PM, Yadav P, Geurts MW. Long-term dosimetric stability of multiple TomoTherapy delivery systems. J Appl Clin Med Phys 2017; 18:137-43. https://doi.org/10.1002/acm2.12085.

4. Miften M, Olch A, Mihailidis D, Moran J, Pawlicki T, Molineu A, et al. Tolerance limits and methodologies for IMRT measurement-based verification QA: recommendations of AAPM Task Group No. 218. Med Phys. 2018:45:e53-83. https://doi.org/10.1002/mp.12810.

5. Sterzing F, Uhl M, Hauswald H, Schubert K, Sroka-Perez G, Chen Y, et al. Dynamic Jaws and dynamic couch in helical tomotherapy. Int J Radiat Oncol Biol Phys. 2010;76:1266-73. https://doi.org/10.1016/j.jprobp.2009. 07.1686.

6. Bresciani S, Dia AD, Maggio A, Cutaia C, Miranti A, Infusino E, et al. Tomotherapy treatment plan quality assurance: the impact of applied criteria on passing rate in gamma index method: gamma passing rate variability. Med Phys. 2013;40:121711. https://doi.org/10.1118/1.4829515.

7. Bresciani S, Miranti A, Di Dia A, Maggio A, Bracco C, Poli M, et al. A pretreatment quality assurance survey on 384 patients treated with helical intensity-modulated radiotherapy. Radiother Oncol. 2016;118:574-6. https://doi.org/10.1016/j.radonc.2015.12.021.

8. Geurts M, Gonzalez J, Serrano-Ojeda P. Longitudinal study using a diode phantom for helical tomotherapy IMRT QA: Iongitudinal study for helical tomotherapy IMRT QA. Med Phys. 2009;36:4977-83. https://doi.org/10. 1118/1.3238153.

9. DiCiccio Thomas J, Bradley Efron. Bootstrap confidence intervals. Stat Sci. 1996;11(3):189-228.

10. Jung $\mathrm{K}$, et al. Comparison of bootstrap confidence interval methods for GSCA using a Monte Carlo simulation. Front Psychol. 2019;10:2215. https://doi.org/10.3389/fpsyg.2019.02215.

11. Kruskal William $\mathrm{H}$, Allen Wallis W. Use of ranks in one-criterion variance analysis. J Am Stat Assoc. 1952;47(260):583-621.

12. Dunn OJ, Clark VA. Applied Statistics: Analysis of Variance and Regression. New York: Wiley, 1974.

13. Jiarpakdee J, Tantithamthavorn C, Hassan AE. The Impact of Correlated Metrics on the Interpretation of Defect Models. in IEEE Transactions on Software Engineering, vol. 47, no. 2, pp. 320-331. https://doi.org/10.1109/ TSE.2019.2891758.

14. Kuhn M, Johnson K. Applied predictive modeling, vol. 26. New York: Springer; 2013.

15. Binny D, Lancaster CM, Byrne M, Kairn T, V. Trapp J, Crowe SB. Tomotherapy treatment site specific planning using statistical process control. Physica Medica 2018; 53:32-9. https://doi.org/10.1016/j.ejmp.2018.08. 003.

16. Yue Q, Duan J, Li R, Yang J. Systematic analysis of the ArcCheck diode arrays for Tomotherapy delivery verification. IJMPCERO. 2014;03:218-25. https://doi.org/10.4236/ijmpcero.2014.34028.

17. Santos T, Ventura T, Mateus J, Capela M, do Carmo Lopes M. On the complexity of helical tomotherapy treatment plans. JACMP 2020; 21:107-118. https://doi.org/10.1002/acm2.12895

18. Binny D, Lancaster CM, Harris S, Sylvander SR. Effects of changing modulation and pitch parameters on tomotherapy delivery quality assurance plans. J Appl Clin Med Phys. 2015;16:87-105. https://doi.org/10.1120/ jacmp.v16i5.5282.

19. Nelms BE, Zhen H, Tomé WA. Per-beam, planar IMRT QA passing rates do not predict clinically relevant patient dose errors: predicative power of conventional IMRT QA. Med Phys. 2011;38:1037-44. https://doi.org/10. $1118 / 1.3544657$. 
20. Hussein M, Adams EJ, Jordan TJ, Clark CH, Nisbet A. A critical evaluation of the PTW 2D-ARRAY seven29 and OCTAVIUS II phantom for IMRT and VMAT verification. J Appl Clin Med Phys. 2013;14:274-92. https://doi.org/ 10.1120/jacmp.v14i6.4460

21. Stasi M, Bresciani S, Miranti A, Maggio A, Sapino V, Gabriele P. Pretreatment patient-specific IMRT quality assurance: a correlation study between gamma index and patient clinical dose volume histogram: correlation between gamma index and dose-volume histogram. Med Phys. 2012;39:7626-34. https://doi.org/10.1118/1.4767763.

\section{Publisher's Note}

Springer Nature remains neutral with regard to jurisdictional claims in published maps and institutional affiliations.
Ready to submit your research? Choose BMC and benefit from:

- fast, convenient online submission

- thorough peer review by experienced researchers in your field

- rapid publication on acceptance

- support for research data, including large and complex data types

- gold Open Access which fosters wider collaboration and increased citations

- maximum visibility for your research: over $100 \mathrm{M}$ website views per year

At BMC, research is always in progress.

Learn more biomedcentral.com/submissions 